\title{
Estudio de oferta y demanda de la guayaba en la región del Río Suárez"
}

\section{Guava's Marketing Research at Suarez River Region.}

\author{
Orlando de Jesús Marín Lorduy², Iván Alfredo Cotes Bruges³, Martha Eugenia \\ Carreño Gualdrón ${ }^{4}$, Alexander Hernández Ríos ${ }^{5}$ \\ Universidad de Santander - UDES, Bucaramanga, Colombia.
}

Artículo recibido en octubre de 2014; artículo aceptado en octubre de 2014.

Citación del artículo: Marín Lorduy, O.; Cotes Bruges, I.; Carreño Gualdrón, M \& Hernández A. (2014). Estudio de oferta y demanda de la guayaba en la región del Río Suárez. I+D Revista de Investigaciones,4(2), 49-58.

\section{Resumen}

La cadena agroalimentaria de la guayaba tiene la gran preocupación de la baja competitividad en la obtención de este fruto(Cadena productiva regional de la guayaba y su industria Boyacá y Santander, 2007), hecho que motiva que los integrantes de la cadena hayan querido desarrollar un estudio de mercados que determine su oferta y demanda actual, el cual se

${ }^{1}$ Artículo empírico de enfoque cuantitativo, resultado de un proyecto de investigación en curso, desarrollado en el grupo de investigación Nuevas tecnologías del programa Ingeniería Industrial, financiado por la Universidad de Santander - UDES de la ciudad de Bucaramanga (Colombia). Dirección Campus Universitario Lagos del Cacique, PBX: 57-7-6516500. Fecha de inicio: Enero 2014, fecha de terminación: Octubre 2014.

${ }^{2}$ Ingeniero Industrial, Universidad Tecnológica de Bolívar. MBA Leader con énfasis en Dirección general de empresas, Instituto Eurotechnology empresas A Coruña. Docente - investigador del grupo: Nuevas tecnologías. Universidad de Santander - UDES de la ciudad de Bucaramanga (Colombia). Dirección Campus Universitario Lagos del Cacique, PBX: 57-7-6516500. Correo electrónico institucional: Orlando.marin@udes.edu.co

${ }^{3}$ Administrador de comercio internacional, Universidad popular del Cesar. Especialista en Administración de recursos informáticos, Universidad de Santander - UDES. Docente - investigador del grupo: Nuevas tecnologías. Universidad de Santander - UDES de la ciudad de Bucaramanga (Colombia). Dirección Campus Universitario Lagos del Cacique, PBX: 57-7-6516500. Correo electrónico institucional: icotes@udes.edu.co

${ }^{4}$ Administradora de Empresas, Universidad Cooperativa de Colombia. Especialista en finanzas, Universidad Autónoma de Bucaramanga. Docente - investigador del grupo: Nuevas tecnologías. Universidad de Santander - UDES de la ciudad de Bucaramanga (Colombia). Dirección Campus Universitario Lagos del Cacique, PBX: 57-7-6516500. Correo electrónico institucional: martha.carreno@udes.edu.co

${ }^{5}$ Estudiante de Ingeniería Industrial, Universidad de Santander - UDES. Dirección Campus Universitario Lagos del Cacique, PBX: 57-7-6516500. Correo electrónico: ingalexanderh@hotmail.com 
presenta en este documento. Dicho estudio se dividió en: (1) un análisis de demanda de la guayaba en fresco, extraído de una encuesta aplicada a una muestra de cuarenta y seis fabricantes de bocadillo de la región, mediante investigación exploratoria descriptiva, y (2) un estudio exploratorio descriptivo de la oferta de esta fruta mediante encuesta sobre asociaciones representantes de cultivadores del fruto. Como principales resultados se describieron las características de comercialización de la pulpa, identificando el volumen de demanda y oferta, se estimó la capacidad de la oferta real para el mercado nacional y la demanda real del mercado nacional e internacional, se identificaron tanto los espacios de mercado en los que existe demanda insatisfecha como los eslabones de la cadena en los canales de la comercialización.

\section{Abstract.}

The food chain of guava has a great concern about the low competitiveness in obtaining this fruit. Therefore, the chain decided to develop a marketing study in order to determine the current supply and demand, which was divided in: (1) a descriptive exploratory study of demand of fresh guava based on a survey that included forty six manufacturers at the region, (2) a descriptive exploratory study of supply, a survey on a sample of associations that represent the farmers of the region. The results highlight the marketing characteristics of the pulp, identifying the volume of demand and supply, the capacity of the real offer for the domestic market, and the real demand of the domestic and international market, also were identified the markets where there is not attended demand, and the links of the chain in marketing channels.Introducción

En Colombia, desde hace más de un siglo se desarrolla la agroindustria de la guayaba, siendo esta un fruto tropical con grandes características como su gran aporte vitamínico, en minerales y fibra, que benefician al hombre en su dieta alimenticia (Castro-Vargas, Rodríguez-Valera, \& Parada-Alfonso, 2010).

La producción de guayaba en Colombia, para el año 2011, ascendió a 128.200 toneladas. De las cuales 63.000 toneladas $(49 \%)$ fueron producidas por el núcleo productivo de Boyacá y Santander, también denominado el eje guayabero de Colombia (Vélez - Santander y Ricaurte-Boyacá o región del Río Suárez). En la actualidad existen 250 fábricas de bocadillo a nivel nacional, de las cuales 131 (52\%) se encuentran en la región objeto de este estudio. La producción se concentra en el departamento de Santander, ya que éste posee más de 15.000 hectáreas cultivadas en la provincia de Vélez dentro de sus 15 municipios. (Cadena agroalimentaria de la guayaba y su industria, 2013).

De otra parte, la cadena agroalimentaria de la Guayaba busca desarrollar proyectos de investigación básica y aplicada, consolidando una oferta de productos potencialmente exportables, con características tradicionales y de impacto en mercados externos, brindando beneficios a los eslabones de la producción, transformación y comercialización. (Ministerio 
de Agricultura y Desarrollo Rural, 2013); esta es una de las razones que ha motivado el trabajo con este gremio por parte de la Universidad de Santander-UDES y de centros de investigación.

El eslabón industrial no escapa de los problemas que afectan la comercialización de la fruta en fresco. Por ejemplo, en la fabricación de bocadillos hay obsolescencia de equipos y por eso se consume mucho combustible, lo que afecta negativamente el medio ambiente; varios fabricantes no poseen infraestructura de almacenamiento adecuada para conservar las pulpas, lo que obliga a la parálisis de la producción y a venderla a más bajo precio; los ingresos de los productores son pequeños, con el consecuente bajo nivel de vida, y en promedio el kilo de la fruta cuesta $\$ 32 \mathrm{COP}$ e incrementa a $\$ 48$ COP cuando la cosecha disminuye.

En un estudio paralelo a este en el que se pretende elaborar un plan de marketing para el bocadillo en la misma región, se preparan iniciativas para el fortalecimiento agroindustrial de dicho producto, para acceder a más mercados tanto en el ámbito nacional como internacional, permitiendo la incursión de la guayaba en la cadena de valor y beneficiando a los municipios productores de la misma.

La guayaba tiene un mercado nacional prácticamente cautivo, ante la inexistencia de oferta de fruta extranjera y una larga tradición regional de consumo del producto. La demanda nacional es muy estable y poco exigente en términos de calidad y variedad, aunque muestra una conducta racional ante el incremento en los precios, lo cual lleva al consumidor a preferir otros frutos como mango, manzana y banano. En aras de mejorar su comercialización, es necesario, además de industrializarla, ofertar sus grandes beneficios y mejorar la cadena de suministro, sin embargo, esto hace parte de un plan de mercadeo posterior.

Es preciso comentar que la guayaba se caracteriza por presentar dos cosechas anuales, una a mitad de año llamada "cosecha de mitaca", y otra a fin de cada año conocida como "cosecha principal". Se puede referenciar que durante 2007 y 2008 sólo se presentó una cosecha anual, mientras que de 2009 en adelante se han presentado producciones continuas con picos correspondientes a las dos cosechas anuales mencionadas. (Solarte, Insuasty, \& Melgarejo, Calendario fenológico de la guayaba en la hoya del río Suarez, 2010).

\section{Tipo de estudio}

El presente estudio es de tipo no experimental ya que se seleccionaron sujetos a quienes se les midieron valores de las variables estudiadas, es decir, asociaciones que representaban a los agricultores de la región, sobre quienes se observó y midió la oferta, y microempresarios fabricantes de bocadillo, en quienes se midió la demanda de esta fruta. El diseño fue descriptivo ya que se concluyó detallando el comportamiento de las variables asociadas a demanda y oferta; por otro lado las respectivas demanda y oferta fueron medidas en un momento estático muy específico de tiempo. 


\section{Participantes}

Para la aplicación de la encuesta de la demanda de la guayaba en fresco se elaboró un cuestionario estructurado, el cual se aplicó directamente a una muestra de cuarenta y seis empresas fabricantes de bocadillo existentes en la región del Río Suárez, durante los meses de junio a agosto de 2014. Por otro lado en el estudio de la oferta, se aplicó igualmente un cuestionario estructurado a una muestra estadísticamente representativa igual a diez asociaciones que representaban a los cultivadores de guayaba ubicados en la misma región. El cuestionario se aplicó durante el mes de junio de 2014.

\section{Materiales e instrumentos}

Se aplicó una primera encuesta buscando medir aspectos de la demanda de la guayaba en fresco a los fabricantes de bocadillo, y una segunda que buscaba medir aspectos de la oferta a las asociaciones representantes de los cultivadores. Los instrumentos aplicados fueron validados mediante aplicación a premuestra de manera que proporcionaron datos confiables.

\section{Encuesta.}

En la encuesta de la oferta se evaluaron las siguientes dimensiones: tipo de cultivo, variedad cultivada de fruto, proveedores de insumos, frecuencia y condiciones de compra de estos. Por otro lado, se recolectó información relacionada con la cantidad de árboles sembrados por hectárea y la edad de los mismos, su producción por hectárea y la frecuencia de producción. Se analizaron los principales compradores, frecuencia y cantidades vendidas, precios y condiciones de venta, y finalmente se indagó sobre las fortalezas y dificultades en la comercialización de la guayaba en fresco.

En la investigación de la demanda se evaluaron las siguientes dimensiones: frecuencia y volumen de compra, características del producto comprado, principales proveedores, costos incurridos en la compra y finalmente los proveedores.

\section{Procedimiento}

Para llevar a cabo la investigación, se describieron inicialmente las características del sector agroindustrial de la guayaba, refiriendo los procesos de comercialización de la pulpa en la región del Río Suárez; luego se estimó la capacidad de la oferta real y la demanda real, identificando posteriormente la demanda insatisfecha y los eslabones de comercialización en la cadena. Se generaron conclusiones válidas para el sector agroindustrial y para la academia.

\section{Resultados \\ Oferta}

En cuanto a la oferta de la guayaba en fresco se hicieron varios hallazgos como:

Solamente un $20 \%$ de los cultivadores tiene siembras exclusivamente silvestres, el resto tiene cultivos combinados, es decir, entre silvestres y tecnificados. La proporción total de los cultivos de la muestra es de un $75 \%$ silvestre y $25 \%$ tecnificado. La oferta reportada por la muestra estudiada (diez asociaciones) se eleva a ciento noventa y siete (197) toneladas semanales, lo cual se extrapola a las trece asociaciones con una 
cifra que supera las doscientos cincuenta y seis $(256,1)$ toneladas semanales. Se encuentra que todos en la región cultivan las variedades de guayaba roja y blanca en igual proporción ( $50 \%$ y $50 \%$ ), y no cultivan otras especies conocidas como la guayaba pera. El cultivo en igual proporción de las dos especies mencionadas (guayaba roja y guayaba blanca) se debe a que los fabricantes de bocadillo requieren para la producción una mezcla igual de las dos especies.

El 90\% de los cultivadores utiliza insumos químicos para el control de plagas, mientras que todos utilizan insumos orgánicos como abono para sus siembras. En este sentido, reportan que el $90 \%$ compran dichos insumos a almacenes agrícolas, mientras que el $10 \%$ los adquiere mediante personas naturales. El $70 \%$ de ellos efectúa la compra de los insumos cada mes, mientras que el resto lo hace cada seis meses. Todos ellos acostumbran a hacer la compra estrictamente de contado.

En referencia a la cantidad de árboles reportados por hectárea en los cultivos de la región, se puede mencionar que en el $43 \%$ de los cultivos tiene menos de 250 árboles por hectárea, el 21\% tiene entre 251 - 300 árboles por cada hectárea sembrada, el 29\% cuenta con un rango de 351 - 400, y sólo el 7\% tiene más de 400 árboles por hectárea.

En cuanto a la edad de los árboles sembrados se reporta que el $90 \%$ de los agricultores cuenta con árboles de entre $2-5$ años de edad, el $70 \%$ de ellos tiene árboles con más de 15 años de edad, el
$20 \%$ de ellos tiene árboles de 6 - 10 años de edad y el $10 \%$ de los cultivadores cuenta con árboles de entre 11 - 15 años. E1 9\% de los cultivadores saca su producción cada tres meses, mientras que la mayoría $(91 \%)$ lo hace cada seis meses.

La mayoría de los cultivadores (90\%) hace control fitosanitario contra plagas así: La totalidad de ellos actúa contra "el picudo", el $11 \%$ contra la mosca de la guayaba, el $78 \%$ contra "el aniñador", el 56\% contra el gusano taladrador, y el 11\% contra "el peta". Los métodos más utilizados para el control del picudo son: embolsado de frutos, eliminación de frutos en mal estado, selección de árboles trampa, desfase de cosecha, uso de químicos y uso de hormigas predadoras (Corpoica, Ica, Gobernación de Santander, 2007).

Los agricultores reciben asesoría y asistencia técnica de diversas entidades como el SENA (40\%), el ICA (10\%) y Exagro (10\%). Otros se asesoran de particulares (60\%) o de las Unidades Municipales de Asistencia Técnica Agropecuaria -Umatas (10\%).

El $100 \%$ de la producción se vende a los fabricantes de bocadillo, efectuando la venta con frecuencia semanal en un $60 \%$ de los casos, y mensual en el $40 \%$ restante. La cantidad de guayaba vendida semanalmente en la mayoría de los casos $(70 \%)$, oscila entre $1-5$ toneladas semanales, el $20 \%$ vende entre 6 y 10 toneladas semanales, mientras que el $10 \%$ vende más de 10 toneladas semanales. El $80 \%$ de los cultivadores realiza su venta de contado, el resto lo hace a 
crédito.

Se reporta que apenas un poco más de la mitad $(53 \%)$ de los cultivadores aprecian como fortaleza la calidad del producto, el resto considera que el hecho de pertenecer al cluster es una gran ventaja. Por otro lado, casi la mitad de ellos (44\%) reporta que la variabilidad del precio es una dificultad en el momento de la comercialización, otros (33\%) consideran que la dificultad consiste en la estacionalidad del producto, algunos (11\%) consideran que las dificultades del transporte son una desventaja, mientras que una cantidad igual (11\%) piensa que la calidad del producto es un inconveniente.

\section{Demanda}

En cuanto a la demanda del producto se hicieron los siguientes hallazgos:

Una pequeña parte $(9 \%)$ de los fabricantes compra la pulpa de la guayaba una vez a la semana; un porcentaje considerable (28\%), hace la compra dos veces a la semana; otro porcentaje parecido (30\%) lo hace tres veces a la semana; un porcentaje menor $(22 \%)$, lo hace cuatro veces a la semana; mientras que un porcentaje pequeño (7\%) ejecuta sus compras cinco veces a la semana, y finalmente solo un 4\% lo hace seis veces a la semana. El volumen de compra semanal de los 46 encuestados suma 655.100 kilos semanales, por lo que extrapolando a la población (131 fabricantes) se considera que la compra semanal es de 1.865.610,87 kilos, es decir, 1.865,6 toneladas semanales. Los fabricantes comentan que compiten diferenciándose en la compra de la guayaba por las siguientes características: pulpa compacta $(19 \%)$, frescura de la fruta (17\%), aroma (13\%), por proceder de la región (10\%), calidad y buen estado $(10 \%)$, color $(8 \%)$, el resto aduce otras características.

Todos los fabricantes encuestados consideran que hay demanda insatisfecha, aduciendo como factores los siguientes: incidencia de los factores fitosanitarios (10\%), largas distancias obligadas por carretera $(40 \%)$, temporalidad del fruto $(40 \%)$, malas vías de acceso ( $8 \%$ ) y desconocimiento de la oferta $(2 \%)$.

Finalmente los encuestados hicieron mención de los principales factores a los que les adjudican los incrementos en los costos incurridos al momento de comprar la pulpa. Las respuestas fueron: baja producción o escasez por temporalidad (37\%), por la calidad del producto (13\%), largas distancias (24\%), transporte (21\%), factores climáticos (3\%), y otros $(2 \%)$.

Sus proveedores de guayaba son campesinos independientes en un $34 \%$, grandes distribuidores en un 59\% y otros intermediarios $(7 \%)$.

\section{Comentarios}

Derivado de este estudio de mercado se pueden concluir aspectos interesantes que arrojarán luz sobre la oferta, la demanda, sus características y que podrán servir tanto a agricultores como a productores y académicos para entender qué ocurre actualmente con estos 
mercados, y qué líneas se pueden potenciar o mejorar en el futuro de cara a lograr interacciones más eficientes entre oferta y demanda.

Se aprecia que casi todos cuidan sus cultivos contra las plagas, adicionalmente incrementan la producción mediante la aplicación de abonos orgánicos, todos preferiblemente obtenidos de almacenes agrícolas cada mes y comprándolos de contado.

De acuerdo con el conocimiento de los cultivadores, el rango de edad más productivo para los árboles de guayaba se encuentra en la mitad de su ciclo de vida, es decir, entre los seis y quince años de edad. Cada árbol da frutos dos veces al año. Sólo el 30\% reporta tener árboles en ese rango de edad, es decir, un alto porcentaje de la cosecha total de la región debe esperar hasta cinco años para entrar en su máximo punto de producción. Una manera de manipular el período de producción es mediante la poda: cuando los árboles comienzan a florecer, a una parte de ellos se les podan las flores impidiendo así que den fruto, así éstos árboles podados florecen tres meses después de la poda, con lo cual se asegura producción cuatro veces al año. Toda la producción se vende a los fabricantes de bocadillo, asegurando así que se mantenga la cadena.

Los cultivadores consideran en su mayoría que las dificultades comerciales de la cadena están en la variabilidad del precio del producto, precisamente causa de otro factor considerado, la estacionalidad del producto, es decir, éste se torna más costoso cuando hay escasez así: cada tonelada de guayaba se vende a $\$ 143.600 \mathrm{COP}$ cuando la producción es abundante, y a $\$ 605.000$ COP cuando la producción es escasa.

Derivado de este estudio se identifican algunas ventajas comerciales como: inexistencia de intermediarios en la venta a fabricantes de bocadillo, experiencia en el manejo del fruto, gran volumen de venta mediante fabricantes de bocadillo, menor esfuerzo en la venta por la misma razón anterior, y oferta de productos de alta calidad con una variedad relativa (guayaba roja y guayaba blanca). También se identifican algunas desventajas comerciales como: en el caso de la venta a comerciantes mayoristas el cultivador debe darse a la tarea de buscar el intermediario que mejor satisfaga sus necesidades; ingresos bajos en época de escasez del producto; inestabilidad de precios; mercado limitado debido a la falta de información acerca del producto; desconocimiento de parte del cliente sobre los beneficios nutricionales del producto.

En los casos en los que los fabricantes de bocadillo no disponen de suficiente pulpa, estos deben recurrir a los mayoristas, quienes traen el producto desde otras zonas del país. La diferencia entre las frecuencias de compra de los fabricantes y las de venta de los cultivadores, radica en que cada fabricante compra a diversos proveedores.

Los factores climáticos que más inciden en la producción son la temperatura y el régimen de lluvias (Solarte, Romero, \& Melgarejo, 2010). 
Finalmente se observa un gran desfase entre las respectivas oferta y demanda proyectadas, es decir, la primera cifra muestra claramente 256,1 toneladas semanales de la fruta en fresco ofrecida a los fabricantes, pero éstos demandan 1.865,6 toneladas semanales, lo que los hace buscar alternativas con grandes distribuidores (mayoristas y cadenas de almacenes) y en otros mercados nacionales de distinta calidad, como la zona del pacífico colombiano, en la que la calidad del fruto es menor. Dado este gran desfase, y vistas las posibilidades de control fitosanitario, de terreno y de incremento de cosechas mediante aplicaciones técnicas, se pretende llevar a cabo una segunda parte de investigación en la que se elaborará un plan de mercadeo para el fruto en fresco producido en la región, de modo que en un mediano plazo se incremente la oferta llegando a satisfacer por completo la demanda local y la nacional, y a un largo plazo se exporte el producto para poder entrar a competir en los mercados internacionales.

\section{Agradecimientos}

Se extiende un agradecimiento especial al gremio agroindustrial de la guayaba en la región del Río Suárez por suministrar información importante para el desarrollo de este estudio, además de información referente a los contactos y bases de datos para la aplicación de los instrumentos. Se agradece al grupo de nuevas tecnologías del programa de Ingeniería
Industrial de la Universidad de Santander UDES por el apoyo académico durante todo el proceso, y a la Universidad de Santander UDES por la financiación necesaria para llevar a cabo el proyecto mediante convocatoria interna de proyectos focalizados 2013 .

\section{Referencias}

Cadena agroalimentaria de la guayaba y su industria. (2013). Agenda de competitividad de la Cadena agroalimentaria de la guayaba y su industria. Santander, Bucaramanga.

Cadena productiva regional de la guayaba y su industria Boyacá y Santander. (2007). Respuesta integral en generación de competitividad. Barbosa.

Castro-Vargas, H., Rodríguez-Valera, L., \& Parada-Alfonso, F. (2010). Guava (Psidium guajava L.) seed oil obtained with a homemade supercritical fluid extraction system using supercritical CO2 and co-solvent. The Journal of supercritical fluids, 238-242.

Corpoica, Ica, Gobernación de Santander. (2007). Manejo integrado del picudo de la guayaba (Conotrachelus psidii Marshall) en Santander. Barbosa: Produmedios.

Fondo Nacional del Ganado, Fedegan. (18 de Enero de 2014). Contexto ganadero. Recuperado el 10 de Agosto de 2014, de En estudio árboles de guayaba ante amenaza de 
$\begin{array}{llllllllll}\mathrm{i} & \mathrm{n} & \mathrm{s} & \mathrm{e} & \mathrm{c} & \mathrm{t} & \mathrm{o} & \mathrm{s} & \text { : } & \text { (Psidium guajava L.) para el fortalecimiento de }\end{array}$ http://www.contextoganadero.com/agricultura/ la cadena productiva. Bogotá: Universidad en-estudio-arboles-de-guayaba-ante-amenazade-insectos Nacional de Colombia.

Solarte, M. E., Romero, H. M., \& Melgarejo,

Ministerio de Agricultura y Desarrollo Rural. (2013). Agenda nacional de $\mathrm{C}+\mathrm{T}+\mathrm{I}$ de la guayaba - Cadena agroalimentaria de la guayaba.

Solarte, M. E., Insuasty, O., \& Melgarejo, L. M. (2010). Calendario fenológico de la guayaba en la hoya del río Suarez. En A. L. Morales, \& L. M. Melgarejo (Edits.), Desarrollo de productos funcionales promisorios a partir de la guayaba
L. M. (2010). Caracterización ecofisiológica de variedades de guayaba por función de uso. En M. E. Solarte, O. Insuasty, \& L. M. Melgarejo (Edits.), Desarrollo de productos funcionales promisorios a partir de la guayaba (Psidium guajava L.) para el fortalecimiento de la cadena productiva. Bogotá: Repositorio institucional UN bdigital. 